\title{
Two new convolutions for the fractional Fourier transform ${ }^{\star}$
}

\author{
P. K. Anh • L. P. Castro • P. T. Thao • \\ N. M. Tuan
}

\begin{abstract}
In this paper we introduce two novel convolutions for the fractional Fourier transforms (FRFT), and prove natural algebraic properties of the corresponding multiplications such as commutativity, associativity and distributivity, which may be useful in signal processing and other types of applications. We analyze a consequent comparison with other known convolutions, and establish a necessary and sufficient conditions for the solvability of associated convolution equations of both the first and second kind in $L^{1}(\mathbb{R})$ and $L^{2}(\mathbb{R})$ spaces. An example satisfying the sufficient and necessary condition for the solvability of the equations is given at the end of the paper.
\end{abstract}

Keywords convolution - convolution theorem · fractional Fourier transform • convolution equation · filtering

MSC 2010 40E99 - 43A32 - 47B15 - 44A20 - 68T37 · $94 \mathrm{~A} 12$

\footnotetext{
* Accepted author's manuscript (AAM) published in [Wireless Personal Communications 92(2) (2017), 623-637] [DOI: 10.1007/s11277-016-3567-3]. The final publication is available at Springer via http://link.springer.com/article/10.1007/s11277-016-3567-3

P. K. Anh

Dept. of Comput. and Appl. Math., College of Science, Vietnam National University, 334 Nguyen Trai str., Thanh Xuan dist. Ha Noi, Viet Nam

E-mail: anhpk@vnu.edu.vn

L. P. Castro

Center for R\&D in Math. and Appl. and the Dept. of Math., University of Aveiro, 3810-193

Aveiro, Portugal

E-mail: castro@ua.pt

P. T. Thao

Dept. of Math., Ha Noi Architectural University, Km 10, Nguyen Trai str., Thanh Xuan dist., Ha Noi, Vietnam

E-mail: phamthao.hau@gmail.com

N. M. Tuan

Dept. of Math., College of Education, Vietnam National University, G7 Build., 144 Xuan

Thuy Rd., Cau Giay dist., Ha Noi, Vietnam

E-mail: nguyentuan@vnu.edu.vn
} 


\section{Introduction}

To the best of our knowledge the fractional Fourier transform (FRFT) was introduced in the mathematical literature as early as 1929. In fact, as about the initial ideas related with FRFT, we may point out the works of N. Wiener in 1929, H. Weyl in 1930, E. U. Condon in 1937, H. Kober in 1939, A. P. Guinand in 1956, A. L. Patterson in 1959, V. Bargmann in 1961, De Bruijn in 1973 and R. S. Khare in 1974, among others. Then, the concept was somehow reinvented by Namias when solving some differential and partial differential equations in quantum mechanics [1] in 1980. Such results were later improved on by McBride and Kerr 2. During the 1990s, a large number of papers appeared in the literature tying the concept of the fractional Fourier operators to many other fields such as signal processing and optics [3, 4, 5, 6, 7, 8, 9, Recently, it has been widely applied e.g. in radar, watermarking, pattern recognition, cryptography, wavelet transforms and neural networks [10,11, 12, 13, 14. It is also clear that the consideration of integral transforms of fractional type opens new possibilities in fractional signal processing analysis [15. In particular, the FRFT may be interpreted as a rotation by an angle in the time-frequency plane or decomposition of the signal in terms of chirps.

Note that in all the time-frequency representations [16,17], one normally uses a plane with two orthogonal axes corresponding to time and frequency. In the classical sense, if we consider a signal to be represented along the time axis and its ordinary Fourier transform to be represented along the frequency axis, then the Fourier transform operator can be visualized as a change in representation of the signal corresponding to a counterclockwise rotation of the axis by an angle $\pi / 2$. That is why two successive rotations of the signal through $\pi / 2$ will result in an inversion of the time axis - which from the mathematical point of view lead us to the inverse of the Fourier transform. Moreover, four successive rotations will leave the signal unaltered since a rotation through $2 \pi$ of the signal should leave the signal unaltered (and from the mathematical viewpoint it means that the Fourier integral operator is indeed an involution of order four). The FRFT is a linear operator that corresponds to the rotation of the signal through an angle which is not a multiple of $\pi / 2$. Instead, as above mentioned, it provides us with a representation of the signal along an axis which makes an angle $\alpha$ with the time axis. That is why now-a-days it is well recognized that FRFT leads to a generalization of time and frequency domains - being therefore very useful in signal analysis and processing. In particular, this obviously yields the possibility of using the FRFT in time-varying signals for which the classical Fourier transform fails to work (cf. also [18, 19, 20, 21, $22,23,24$ ).

The present paper has the same spirit of the five papers listed below along the time axis: Almeida [25, Zayed [24, Deng et al. [26], Wei at al. 23], and the updated paper of Singh at al. 22, where the formulas for the FRFT's of a product and of a convolution of two functions were introduced in certain function spaces. Those convolutions are very interesting, and applicable to both theoretical and practical problems as they may be viewed as extensions of the 
convolution theorem of the Fourier transform. Namely, a convolution transform, mathematically, is diagonalized by another transform; and in the new (momentum) representation a convolution turns into an operator of multiplication by a function (see [27, 28]). An interesting description of the history of the development of convolutions for FRFT and their potential applications was addressed in 22. We can say that there were many endeavors of researchers, explicit and implicit, of developing this research direction. However, convolutions and products of FRFT have not been studied intensively as those of Fourier transform, because, in our opinion, the FRFT is actually much more complicated than the Fourier one.

The main purpose of this paper is to present two new convolutions for the FRFT, analyze a consequent comparison with other known convolutions, and to establish the solvability of their associated convolution equations of both the first and second kind in $L^{1}(\mathbb{R})$ and $L^{2}(\mathbb{R})$ spaces. At the same time, the paper shows that the convolutions given in [22,23, 24, 25, 26] can be defined in both those spaces. In particular, this will be a key point for the circumstance that the convolution integral equations induced by those convolutions can be solved completely.

The paper is divided into four sections and organized as follows. In the next section, we recall the FRFT, define a $L^{1}$-norm, and present our comments and comprehensive analysis on the convolution and product theorems of the five papers cited above. In Section 3 we give two new convolution multiplications and prove their fundamental properties. As we shall verify, there are two different ways of convoluting in each one of the convolutions. This fact may have some advantage over others in filtering. Indeed, associated with the computational complexity and input conditions, we will have two options for choosing filtering (in which the first possibility may be better than the second one or vice-versa). In Section 4 , by using the mentioned convolutions, we investigate classes of convolution integral equations in $L^{1}(\mathbb{R})$ and deduce their solvability together with explicit solution formulas. We observe that although the results are formulated for objects in $L^{1}(\mathbb{R})$, they still hold true for those in $L^{2}(\mathbb{R})$ as the fractional Fourier operator can be defined in this domain, and the proofs are quite similar. Furthermore, we provide an example of convolution equation which satisfies all the conditions of Theorems 7 and 8 below.

\section{Convolution and product theorems}

This section presents the fractional Fourier transform (together with some necessary notations), shows a slight difference between the convolution and product theorems, and analyzes the well-known convolutions and products associated with FRFT.

The fractional Fourier transform (FRFT) with angle $\alpha$ is defined in $L^{1}(\mathbb{R})$ with the help of the transformation kernel $K_{\alpha}$ and given by

$$
\mathcal{F}_{\alpha}[f](p)=\int_{-\infty}^{\infty} f(x) K_{\alpha}(x, p) d x
$$


where

$$
K_{\alpha}(x, p)= \begin{cases}\frac{c(\alpha)}{\sqrt{2 \pi}} \exp \left\{i a(\alpha)\left(x^{2}+p^{2}-2 b(\alpha) x p\right)\right\} \\ \text { if } \alpha \text { is not a multiple of } \pi \\ \delta(x-p), & \text { if } \alpha \text { is a multiple of } 2 \pi \\ \delta(x+p), & \text { if } \alpha+\pi \text { is a multiple of } 2 \pi,\end{cases}
$$

with

$$
a(\alpha)=\frac{\cot \alpha}{2}, \quad b(\alpha)=\sec \alpha, \quad c(\alpha)=\sqrt{1-i \cot \alpha}
$$

Throughout this paper the constants $a(\alpha), b(\alpha)$ and $c(\alpha)$, for simplicity, will be denoted as $a, b$ and $c$. For $\alpha \in 2 \pi \mathbb{Z}$, the FRFT becomes the identity, and for $\alpha+\pi \in 2 \pi \mathbb{Z}$, it is the parity operator. Therefore, from now on we shall confine our attention to $F_{\alpha}$ for $\alpha \notin \pi \mathbb{Z}$.

In the sequel, we define the norm $\|f\|_{0}$ of $f \in L^{1}(\mathbb{R})$ as

$$
\|f\|_{0}:=\frac{1}{\sqrt{2 \pi|\sin \alpha|}} \int_{\mathbb{R}}|f(x)| d x .
$$

Before going to the next section, we shall analyze and compare the convolutions studied in $[22,23,24,25,26$. Let $F$ denote the Fourier transform defined as

$$
F[f](x)=\int_{-\infty}^{\infty} e^{-i x y} f(y) d y .
$$

Let us use $\mathcal{W}:=F\left(L^{1}(\mathbb{R})\right)$ to denote the Wiener algebra. When comparing in detail the proofs of the convolution theorems of Almeida and others, we observe that the domain $\mathcal{W} \cap L^{1}(\mathbb{R})$ (or $L^{2}(\mathbb{R})$ ) is necessary in the proofs of 25], while the wider domain $L^{1}(\mathbb{R})\left(\right.$ or $\left.L^{2}(\mathbb{R})\right)$ is possible to be considered in other works. In particular, we remark that:

- Equations (2), (4), and (8) in [25] can be considered as convolution theorems in some special circumstance, and they become classical convolution theorems for the Fourier transform when $\alpha=\pi / 2$ (and not as noted in [22]). The reader may refer to [29, Theorem 7.8] formulated in the Schwartz space, which is dense in both the spaces $L^{1}(\mathbb{R})$ and $L^{2}(\mathbb{R})$. For instance, consider the expression (2) in 25] for $\alpha=\pi / 2$, and $z=x y$. Since $x, y \in \mathcal{W} \cap L^{1}(\mathbb{R})$, there exist $x_{0}, y_{0} \in L^{1}(\mathbb{R})$, such that $F x_{0}=x, F y_{0}=y$. We then have $z=F x_{0} \cdot F y_{0}=F\left(x_{0} * y_{0}\right)$. It is easy to show that if $f \in \mathcal{W} \cap L^{1}(\mathbb{R})$, then $\left(F^{2} f\right)(u)=f(-u):=\check{f}(u)$ for almost every $u \in \mathbb{R}$ (with Lebesgue measure). Hence, $(F z)(u)=F^{2}\left(x_{0} * y_{0}\right)(u)=\left(x_{0} * y_{0}\right)(-u)$. Thus,

$$
Z_{\pi / 2}(u)=(F z)(u)=\left(x_{0} * y_{0}\right)(-u)
$$

can be viewed as a convolution (with reflection) despite the implicit form of this formula. The right-hand side of the last identity is exactly as (cf. 
[25, (2)])

$$
\begin{aligned}
(F z)(u) & =\left(x_{0} * y_{0}\right)(-u)=\left(\check{x}_{0} * \check{y}_{0}\right)(u) \\
& =\left(F^{2} x_{0} * F^{2} y_{0}\right)(u)=(F x * F y)(u) .
\end{aligned}
$$

However, without the assumption $x, y \in \mathcal{W} \cap L^{1}(\mathbb{R})$, the expressions (2), (4), and (8) in [25] could not be product identities as the expression $F^{2} x$ may have no sense. Of course, the convolution and product theorems in 25] are still valid for $x, y \in L^{2}(\mathbb{R})$. In general, the three above-mentioned expressions are product identities for $\alpha \in \mathbb{R}$.

- As is showed, the operations $\star$ and $\otimes$ in [24] are convolutions. From our point of view, they are not so cumbersome and may be useful in applications.

- The first expression in [26] is a convolution, and the second one is simply a product identity. However, when $\alpha=\pi / 2$ the second one turns out to be the Fourier case as showed above in Almeida's case.

- Equations (16) and (17) in [23, Theorem 1] are in fact generalized convolution and product theorems (see [27,28]). In this work, the authors use the linear canonical transform (LCT) which is a result of parameterizing the kernel of FRFT by four items. LCTs are general transforms that have many potential applications due to their flexibility. On the other hand, the computation of LCTs may be more expensive since they contain four parameters.

- Finally, the expressions given in [22, (11), and (22)] are updated generalized convolution and product transforms. It should be emphasized that if $x, y \in L^{1}(\mathbb{R})$, then formula (22) may fail due to the fact that the function $z(t)$ defined as in $[22$, (11), (12)] may not be integrable. However, the assumption that $x, y \in \mathcal{W} \cap L^{1}(\mathbb{R})$ guarantees the validity of this theorem, and the expression given in [22, (22)] turns into the Fourier case when $\alpha=\pi / 2-$ as the authors stated there.

Observe that the above-mentioned convolutions and products hold in the Hilbert space $L^{2}(\mathbb{R})$ without any additional condition.

\section{New convolutions and their properties}

In this section, we introduce two new convolutions associated with the FRFT, which are defined in the both domains $L^{1}(\mathbb{R})$ and $L^{2}(\mathbb{R})$, and prove their basic properties. However, only the proofs for the convolutions (3.1) and (3.4) in $L^{1}(\mathbb{R})$ are given, since the other cases can be considered in a similar way.

Definition 1 We define the convolution operation $\odot$ by

$$
\begin{aligned}
h(s):=(f \odot g)(s)=\frac{c}{\sqrt{2 \pi}} \int_{-\infty}^{\infty} e^{i a\left(2 u^{2}-2 s u+\frac{s}{a b}-\frac{u}{a b}\right)} f(u) \times & \\
& g\left(s-u+\frac{1}{2 a b}\right) d u .
\end{aligned}
$$


Theorem 1 Let $\psi(x):=e^{i\left(x-a x^{2}\right)}$. If $f, g \in L^{1}(\mathbb{R})$, then

$$
\begin{aligned}
\|f \odot g\|_{0} & \leq\|f\|_{0}\|g\|_{0}, \\
\mathcal{F}_{\alpha}[f \odot g](x) & =\psi(x) \mathcal{F}_{\alpha}[f](x) \mathcal{F}_{\alpha}[g](x) .
\end{aligned}
$$

In other words, the product $f \odot g$ defines a function belonging to $L^{1}(\mathbb{R})$, and satisfies the convolution theorem for the FRFT associated with the function $\psi$.

Proof We first prove inequality (3.2). Note that $|c|=1 /|\sin \alpha|$. Using $f, g \in$ $L^{1}(\mathbb{R})$, and changing the variable $s-u+1 / 2 a b=v$, we have

$$
\begin{aligned}
& \|f \odot g\|_{0}=\frac{1}{\sqrt{2 \pi|\sin \alpha|}} \int_{-\infty}^{+\infty}|(f \odot g)(s)| d s \\
& \leq \frac{1}{2 \pi|\sin \alpha|} \int_{-\infty}^{+\infty} \int_{-\infty}^{+\infty}|f(u)|\left|g\left(s-u+\frac{1}{2 a b}\right)\right| d u d s \\
& =\frac{1}{2 \pi|\sin \alpha|} \int_{-\infty}^{+\infty}|f(u)| d u \times \int_{-\infty}^{+\infty}|g(v)| d v \\
& =\|f\|_{0}\|g\|_{0},
\end{aligned}
$$

which proves the inequality (3.2). This inequality ensures immediately that the convolution defined by (3.1) belongs to $L^{1}(\mathbb{R})$.

Now we will prove the factorization property (3.3). From the definition (2.1) of FRFT, we have

$$
\begin{aligned}
& \psi(x) \mathcal{F}_{\alpha}[f](x) \mathcal{F}_{\alpha}[g](x) \\
& =e^{i\left(x-a x^{2}\right)} \times \frac{c}{\sqrt{2 \pi}} \int_{-\infty}^{\infty} e^{i a\left(x^{2}+u^{2}-2 x u b\right)} f(u) d u \\
& \quad \times \frac{c}{\sqrt{2 \pi}} \int_{-\infty}^{\infty} e^{i a\left(x^{2}+v^{2}-2 x v b\right)} g(v) d v \\
& =e^{i\left(x-a x^{2}\right)} \times \\
& \quad \frac{c^{2}}{2 \pi} \int_{-\infty}^{+\infty} \int_{-\infty}^{\infty} e^{i a\left[2 x^{2}+u^{2}+v^{2}-2 x b(u+v)\right]} f(u) g(v) d u d v \\
& =\frac{c^{2}}{2 \pi} \int_{-\infty}^{+\infty} \int_{-\infty}^{\infty} e^{i a\left[x^{2}+u^{2}+v^{2}-2 x b\left(u+v-\frac{1}{2 a b}\right)\right]} f(u) g(v) d u d v .
\end{aligned}
$$

Making the change of variables $u=u$ and $s=u+v-\frac{1}{2 a b}$, we obtain

$$
\begin{gathered}
\psi(x) \mathcal{F}_{\alpha}[f](x) \mathcal{F}_{\alpha}[g](x) \\
=\frac{c^{2}}{2 \pi} \int_{-\infty}^{+\infty} \int_{-\infty}^{\infty} e^{i a\left[x^{2}+u^{2}+\left(s-u+\frac{1}{2 a b}\right)^{2}-2 x b s\right]} f(u) \times \\
g\left(s-u+\frac{1}{2 a b}\right) d u d s
\end{gathered}
$$




$$
\begin{aligned}
& =\frac{c^{2}}{2 \pi} \int_{-\infty}^{+\infty} \int_{-\infty}^{\infty} e^{i a\left[x^{2}+2 u^{2}+s^{2}-2 s u+\frac{s}{a b}-\frac{u}{a b}-2 x b s\right]} f(u) \times \\
& g\left(s-u+\frac{1}{2 a b}\right) d u d s \\
& =\frac{c}{\sqrt{2 \pi}} \int_{-\infty}^{+\infty} e^{i a\left[x^{2}+s^{2}-2 x b s\right]} \times \\
& \left\{\frac{c}{\sqrt{2 \pi}} \int_{-\infty}^{\infty} e^{i a\left[2 u^{2}-2 s u+\frac{s}{a b}-\frac{u}{a b}\right]} f(u) g\left(s-u+\frac{1}{2 a b}\right) d u\right\} d s \\
& =\mathcal{F}_{\alpha}\left\{\frac{c}{\sqrt{2 \pi}} \int_{-\infty}^{\infty} e^{i a\left[2 u^{2}-2 s u+\frac{s}{a b}-\frac{u}{a b}\right]} f(u) \times\right. \\
& \left.g\left(s-u+\frac{1}{2 a b}\right) d u\right\}(x)=\mathcal{F}_{\alpha}[f \odot g](x) .
\end{aligned}
$$

The proof is complete.

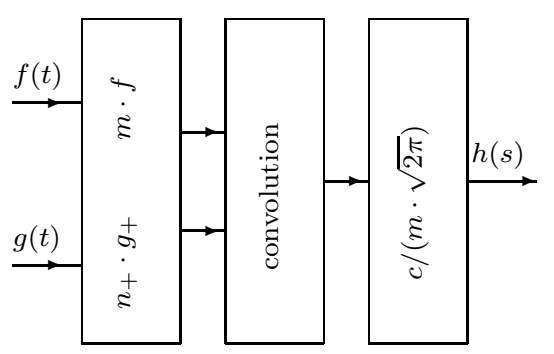

Fig. 1 First way of performing the convolution 3.1

Let us write

$$
m(t):=e^{i a t^{2}}, \quad n_{ \pm}(t):=e^{i a\left(t^{2} \pm \frac{1}{a b} t\right)}
$$

and take into account

$$
g_{ \pm}(t):=g\left(t \pm \frac{1}{a b}\right)
$$

in which $g_{ \pm}$can be considered as a delay or shift of the function $g$ with the step $(1 / a b)$. Clearly, the functions $m$ and $n_{ \pm}$have no zeros and they have equal constant magnitude, i.e., $|m(t)|=\left|n_{ \pm}(t)\right|=1$. Therefore, we can write

$$
m^{-1}(t):=\frac{1}{m(t)}, n_{ \pm}^{-1}(t):=\frac{1}{n_{ \pm}(t)} .
$$

There are two different ways of performing the convolution (3.1) via the Fourier convolution denoted by $*$, as it will be explained below. 
(1) We can represent $h(s):=(f \odot g)(s)$ as

$$
h(s)=(m \cdot f) *\left(n_{+} \cdot g_{+}\right)(s) \cdot \frac{1}{m(s)} \cdot \frac{c}{\sqrt{2 \pi}} .
$$

In this case, the convolution of $f$ and $g$ is obtained by multiplying $f$ by a chirp $(m)$, convolving with $g$ delayed by $(1 / a b)$ and multiplied by a new chirp $\left(n_{+}\right)$, dividing by a chirp $(m)$ and scaling by a factor $(c / \sqrt{2 \pi})$.

(2) On the other hand, we can write

$$
h(s)=\left(n_{-} \cdot f\right) *\left(m \cdot g_{+}\right)(s) \cdot \frac{1}{n_{-}(s)} \cdot \frac{c}{\sqrt{2 \pi}} .
$$

Then the same convolution of $f$ and $g$ is obtained by multiplying $f$ by a chirp $\left(n_{-}\right)$, convolving with $g$ delayed by $(1 / a b)$ and multiplied by a different chirp $m$, dividing by the chirp $n_{-}$and scaling by a factor $(c / \sqrt{2 \pi})$.

Therefore, there are also two options for choosing chirp functions. This fact can be useful for comparison realizable approaches and (numerical) solutions for practical problems. Nevertheless, the FRFT of this convolution is the same as in the expression on the right-hand side of (3.3). Figures 1 and 2 illustrate two different ways of performing the convolution.

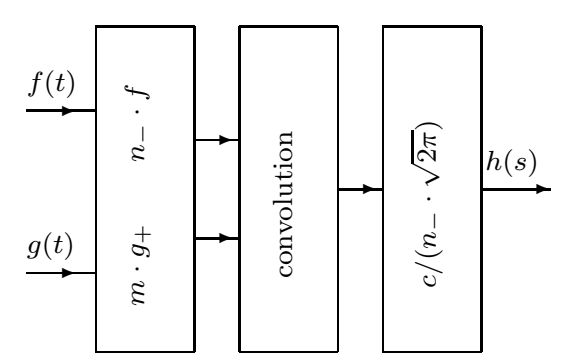

Fig. 2 Second way of performing the convolution 3.1

In other words, convolution (3.1), when applied to some specific problems, is more flexible than those in [22, 23, 24, 25, 26.

As we shall verify in what follows, convolution (3.1) satisfies the commutative, associative and distributive properties:

- Commutativity. From the factorization property (3.3), we have

$$
\begin{aligned}
& \mathcal{F}_{\alpha}[f \odot g](x)=\psi(x) \mathcal{F}_{\alpha}[f](x) \mathcal{F}_{\alpha}[g](x), \\
& \mathcal{F}_{\alpha}[g \odot f](x)=\psi(x) \mathcal{F}_{\alpha}[f](x) \mathcal{F}_{\alpha}[g](x),
\end{aligned}
$$

which implies that

$$
\mathcal{F}_{\alpha}[f \odot g](x)=\mathcal{F}_{\alpha}[g \odot f](x) .
$$

Hence $f \odot g=g \odot f$. 
- Associativity. From the factorization property (3.3), we have

$$
\begin{aligned}
& \mathcal{F}_{\alpha}[(f \odot g) \odot h](x)=\psi^{2}(x) \mathcal{F}_{\alpha}[f](x) \mathcal{F}_{\alpha}[g](x) \mathcal{F}_{\alpha}[h](x), \\
& \mathcal{F}_{\alpha}[f \odot(g \odot h)](x)=\psi^{2}(x) \mathcal{F}_{\alpha}[f](x) \mathcal{F}_{\alpha}[g](x) \mathcal{F}_{\alpha}[h](x),
\end{aligned}
$$

which implies that

$$
\mathcal{F}_{\alpha}[(f \odot g) \odot h](x)=\mathcal{F}_{\alpha}[f \odot(g \odot h)](x) .
$$

Hence,

$$
(f \odot g) \odot h=f \odot(g \odot h) .
$$

- Distributivity. Observing that

$$
\mathcal{F}_{\alpha}[f \odot(g+h)](x)=\psi(x) \mathcal{F}_{\alpha}[f](x) \mathcal{F}_{\alpha}[g+h](x),
$$

and

$$
\begin{aligned}
\mathcal{F}_{\alpha}[f \odot g+f \odot h] & (x) \\
& =\psi(x) \mathcal{F}_{\alpha}[f](x) \mathcal{F}_{\alpha}[g](x)+\psi(x) \mathcal{F}_{\alpha}[f](x) \mathcal{F}_{\alpha}[h](x)
\end{aligned}
$$

we get

$$
\mathcal{F}_{\alpha}[f \odot(g+h)](x)=\mathcal{F}_{\alpha}[f \odot g+f \odot h](x) .
$$

Hence,

$$
f \odot(g+h)=f \odot g+f \odot h .
$$

Definition 2 We define the product $f \otimes g$ by

$$
\begin{aligned}
h(s):=(f \otimes g)(s)=\frac{c}{\sqrt{2 \pi}} \int_{-\infty}^{\infty} e^{i a\left(2 u^{2}-2 s u-\frac{s}{a b}+\frac{u}{a b}\right)_{\times}} & \\
& f(u) g\left(s-u-\frac{1}{2 a b}\right) d u .
\end{aligned}
$$

The following theorem is proved similarly to Theorem 1

Theorem 2 Let $\zeta(x)=e^{i\left(-x-a x^{2}\right)}$. If $f, g \in L^{1}(\mathbb{R})$, then:

$$
\begin{aligned}
\|f \otimes g\|_{0} & \leq\|f\|_{0}\|g\|_{0}, \\
\mathcal{F}_{\alpha}[f \otimes g](x) & =\zeta(x) \mathcal{F}_{\alpha}[f](x) \mathcal{F}_{\alpha}[g](x) .
\end{aligned}
$$

In other words, the product $f \otimes g$ defines a function belonging to $L^{1}(\mathbb{R})$, and satisfies the convolution theorem for the FRFT associated with the function $\zeta$.

Similarly to the convolution (3.1), there are also two different ways of performing the convolution (3.4). Namely:

(1) $h(s)=(m \cdot f) *\left(n_{+} \cdot g_{-}\right)(s) \cdot m^{-1}(s) \cdot(c / \sqrt{2 \pi})$;

(2) $h(s)=\left(n_{+} \cdot f\right) *\left(m \cdot g_{-}\right)(s) \cdot n_{+}^{-1}(s) \cdot(c / \sqrt{2 \pi})$.

We will omit the corresponding illustrative figures due to limitations of space. 
Remark 1 The convolution (3.4) also satisfies the commutative, associative and distributive properties. Let us omit the proofs for this claim as they are similar to those of convolution (3.1).

Thanks to inequalities (3.2) and (3.5), the convolution operators defined by (3.1) and (3.4) are bounded in $L^{1}(\mathbb{R})$. From an algebraic point of view, the space $L^{1}(\mathbb{R})$, equipped with each one of the convolution multiplications (3.1) and (3.4), becomes a commutative Banach algebra.

\section{Classes of convolution equations}

In this section, we establish the solvability of several classes of convolution equations associated with the FRFT, and obtain their explicit solutions formulas.

We start by considering the following type of integral equation in the Banach space $L^{1}(\mathbb{R})$ :

$$
\lambda \varphi(s)+(k \odot \varphi)(s)=f(x),
$$

where $\lambda \in \mathbb{C}$ and $k \in L^{1}(\mathbb{R})$ are given, and $\varphi$ will be determined in this space. We shall use the notation

$$
A(s):=\lambda+\psi(s) \mathcal{F}_{\alpha}[k](s) .
$$

The following proposition is useful for proving Theorem 3 .

Proposition 1 (1) If $\lambda \neq 0$, then $A(s) \neq 0$ for every $s$ outside a finite interval.

(2) If $A(s) \neq 0$ for every $s \in \mathbb{R}$, then the function $1 / A(x)$ is bounded and continuous on $\mathbb{R}$.

Proof (1) By the Riemann-Lebesgue lemma, the function $A(x)$ is continuous on $\mathbb{R}$ and

$$
\lim _{|x| \rightarrow \infty} A(x)=\lambda \neq 0,
$$

i.e., $A(x)$ takes the value $\lambda$ at infinity. Since $\lambda \neq 0$ and $A(x)$ is continuous, there exists an $R>0$ such that $A(x) \neq 0$ for every $|x|>R$. Item (1) is proved.

(2) Due to the continuity of $A$ and $\lim _{|s| \rightarrow \infty} A(s)=\lambda \neq 0$, there exist $R_{0}>0, \epsilon_{1}>0$ such that

$$
\inf _{|x|>R_{0}}|A(x)|>\epsilon_{1}
$$

As $A$ is continuous and does not vanish on the compact set

$$
S\left(0, R_{0}\right)=\left\{x \in \mathbb{R}:|s| \leq R_{0}\right\},
$$

there exists $\epsilon_{2}>0$ such that

$$
\inf _{|s| \leq R_{0}}|A(s)|>\epsilon_{2}
$$


We deduce

$$
\sup _{s \in \mathbb{R}} \frac{1}{|A(s)|} \leq \max \left\{\frac{1}{\epsilon_{1}}, \frac{1}{\epsilon_{2}}\right\}<\infty .
$$

This implies that the function $1 /|A(s)|$ is continuous and bounded on $\mathbb{R}$. Since $\mathcal{F}_{\alpha} f \in L^{1}(\mathbb{R})$, we have $\left(\mathcal{F}_{\alpha} f / A\right) \in L^{1}(\mathbb{R})$. The proposition is proved.

Theorem 3 Assume that $A(s) \neq 0$ for every $s \in \mathbb{R}$, and one of the following conditions is satisfied:

(i) $\lambda \neq 0$, and $\mathcal{F}_{\alpha}[f] \in L^{1}(\mathbb{R})$;

(ii) $\lambda=0$, and $\frac{\mathcal{F}_{\alpha} f}{\mathcal{F}_{\alpha} k} \in L^{1}(\mathbb{R})$.

Then equation (4.1) has a solution in $L^{1}(\mathbb{R})$ if and only if

$$
\mathcal{F}_{-\alpha}\left(\mathcal{F}_{\alpha} f / A\right) \in L^{1}(\mathbb{R}) .
$$

If this is the case, then the solution is given by

$$
\varphi=\mathcal{F}_{-\alpha}\left(\mathcal{F}_{\alpha} f / A\right) .
$$

Proof Let us first assume that (i) is fulfilled.

Necessity. Suppose that equation (4.1) has a solution $\varphi \in L^{1}(\mathbb{R})$. Applying $\mathcal{F}_{\alpha}$ to both sides of equation (4.1) and using the factorization identity in Theorem 1. we obtain

$$
A(s)\left(\mathcal{F}_{\alpha} \varphi\right)(s)=\left(\mathcal{F}_{\alpha} f\right)(s) .
$$

Since $A(s) \neq 0$ for every $s \in \mathbb{R}$,

$$
\mathcal{F}_{\alpha} \varphi=\frac{\mathcal{F}_{\alpha} f}{A} .
$$

As the function $1 / A(x)$ is bounded and continuous on $\mathbb{R}$ (cf. Proposition 1) and $\mathcal{F}_{\alpha} f \in L^{1}(\mathbb{R})$, we deduce that $\left(\mathcal{F}_{\alpha} f / A\right) \in L^{1}(\mathbb{R})$. We can now apply the inverse transform of $\mathcal{F}_{\alpha}$ to (4.2) to obtain the solution as stated in the theorem. The necessity part is proved.

Sufficiency. Consider the function

$$
\varphi:=\mathcal{F}_{-\alpha}\left(\frac{\mathcal{F}_{\alpha} f}{A}\right) .
$$

It implies that $\varphi \in L^{1}(\mathbb{R})$. Hence, $\mathcal{F}_{\alpha} \varphi=\mathcal{F}_{\alpha} f / A$. Equivalently, $A\left(\mathcal{F}_{\alpha} \varphi\right)=$ $\mathcal{F}_{\alpha} f$. Due to the factorization identity,

$$
\mathcal{F}_{\alpha}[\lambda \varphi+(k \odot \varphi)]=\mathcal{F}_{\alpha} f .
$$

By the uniqueness theorem of $\mathcal{F}_{\alpha}$, we conclude that $\varphi$ fulfills equation (4.1) for almost every $s \in \mathbb{R}$. Item (i) is proved.

Since $|\psi(x)|=1$, the function $1 / \psi$ is continuous and bounded on $\mathbb{R}$. Hence, $\mathcal{F}_{\alpha} f / \mathcal{F}_{\alpha} k \in L^{1}(\mathbb{R})$ if and only if $\mathcal{F}_{\alpha} f /\left(\psi \cdot \mathcal{F}_{\alpha} k\right) \in L^{1}(\mathbb{R})$. Therefore, the case of (ii) may be proved similarly to that of item (i). The proof of Theorem 3 is complete. 
Observe that in the last theorem we have just analyzed both situations, where (4.1) can be a first or second kind integral equation depending whether $\lambda=0$ or $\lambda \neq 0$, respectively.

Theorem 4 below can be proved in the same way as Theorem 3 .

Theorem 4 Assume that

$$
B(s):=\lambda+\zeta(s) \mathcal{F}_{\alpha}[k](s) \neq 0
$$

for every $s \in \mathbb{R}$, and that one of the following conditions is satisfied:

(i) $\lambda \neq 0$, and $\mathcal{F}_{\alpha}[f] \in L^{1}(\mathbb{R})$;

(ii) $\lambda=0$, and $\frac{\mathcal{F}_{\alpha} f}{\mathcal{F}_{\alpha} k} \in L^{1}(\mathbb{R})$.

Then, the equation

$$
\lambda \varphi(s)+(k \otimes \varphi)(s)=f(x)
$$

has a solution in $L^{1}(\mathbb{R})$ if and only if $\mathcal{F}_{-\alpha}\left(\mathcal{F}_{\alpha} f / B\right) \in L^{1}(\mathbb{R})$. If this is the case, then the solution is given by

$$
\varphi=\mathcal{F}_{-\alpha}\left(\mathcal{F}_{\alpha} f / B\right) .
$$

We can solve the convolution equations induced by the convolutions given in the works $22,23,24,26$. Namely, let us use the common symbols $\star$ and $\theta(x)$ to denote the convolution operations and the weight-functions given in those papers, respectively. Consider the following equation:

$$
\lambda \varphi(s)+(k \star \varphi)(s)=f(s)
$$

where $\lambda \in \mathbb{C}$ and $k \in L^{1}(\mathbb{R})$ are given, and $\varphi$ is to be found in this space. We set

$$
C(s):=\lambda+\theta(s) \mathcal{F}_{\alpha}[k](s) .
$$

Theorem 5 Assume that $C(s) \neq 0$ for every $s \in \mathbb{R}$, and that one of the following conditions holds true:

(i) $\lambda \neq 0$, and $\mathcal{F}_{\alpha}[f] \in L^{1}(\mathbb{R})$;

(ii) $\lambda=0$, and $\frac{\mathcal{F}_{\alpha} f}{\mathcal{F}_{\alpha} k} \in L^{1}(\mathbb{R})$.

Then equation (4.3) has a solution in $L^{1}(\mathbb{R})$ if and only if

$$
\mathcal{F}_{-\alpha}\left(\mathcal{F}_{\alpha} f / C\right) \in L^{1}(\mathbb{R}) .
$$

If this is the case, then the solution is given by

$$
\varphi=\mathcal{F}_{-\alpha}\left(\mathcal{F}_{\alpha} f / C\right) .
$$

The proof of this theorem is in the same way as that of Theorem 3. and hence is here omitted. 
Example 1 The following equation can serve as an illustration of the abovementioned theorems including the convolutions considered in the four papers just cited above. It suffices to formulate the results for the case of $L^{1}(\mathbb{R})$ as those for $L^{2}(\mathbb{R})$ are similar. Consider the convolution equation

$$
\lambda \varphi(x)+(k \star \varphi)(x)=f(x),
$$

for any $\lambda \in \mathbb{C}$, and the symbol $\star$ denotes any convolution multiplication among (3.1), (3.4) and those in [22,23,24,26]. We choose $k(x)=e^{-a|x|}$ with $\Re(a)>0$, $f(x)=e^{-\frac{1}{2} x^{2}}$. It is easily seen that $k, f \in L^{1}(\mathbb{R})$. Let us denote by $K_{\alpha}(x)$ the FRFT of $k$. Obviously, $|\theta(x)|=1$, and for a fixed $\lambda$ the function

$$
M(x)=\lambda+\theta(x) K_{\alpha}(x)
$$

is bounded and continuous, and tends to $\lambda$ as $|x| \rightarrow+\infty$.

- The case $\lambda \neq 0$. It holds $K_{\alpha} \in L^{1}(\mathbb{R})$. Additionally, note that the function $\theta(x) K_{\alpha}(x)$ is continuous and bounded, and vanishing at infinity. Therefore, if $\lambda$ is arbitrarily and sufficiently large, then $M(x) \neq 0$ for every $x$. For example, the assumption that $|\lambda|>\max _{x \in \mathbb{R}}\left|\theta(x) K_{\alpha}(x)\right|$ is a sufficient condition which guarantees that $M(x)$ is a non-vanishing function. Concerning the second assumption, we have

$$
\mathcal{F}_{\alpha}[f](x)=e^{-\frac{1}{2} x^{2}} \in L^{1}(\mathbb{R}) .
$$

Therefore, we have obtained the solvability of the equation for this case, and we can give its explicit solution.

- The case $\lambda=0$. We can prove without difficulty that $\mathcal{F}_{\alpha}[f] / \mathcal{F}_{\alpha}[k] \in L^{1}(\mathbb{R})$. For instance, if it is the Fourier case, then

$$
F[f](s) / F[k](s)=2 a\left(a^{2}+s^{2}\right) e^{-\frac{1}{2} s^{2}} .
$$

This function belongs to $L^{1}(\mathbb{R})$, and it therefore fulfills the condition in Theorems 3 and 4 .

Thus, in both cases all the conditions of Theorems 3 and 4 are fulfilled, hence the corresponding equation possesses a solution and we have the explicit solution formula.

Conclusion. We have introduced two new convolutions associated with the FRFT, and established the complete solvability of the convolution equations induced by these convolutions. Observe that the explicit solution formula was proved for the above-mentioned convolution-type equations, which may be of the first or the second kind integral equations.

Acknowledgements P. K. Anh, P. T. Thao, and N. M. Tuan were partially supported by the Viet Nam National Foundation for Science and Technology Development (NAFOSTED). L. P. Castro was supported in part by the Portuguese Foundation for Science and Technology ("FCT-Fundação para a Ciência e a Tecnologia"), through CIDMA - Center for Research and Development in Mathematics and Applications, within project UID/MAT/04106/2013. 


\section{References}

1. Namias, V. (1980). The fractional Fourier transform and its application to quantum mechanics. IMA. J. Appl. Math., 25(3), 241-265.

2. McBride, A.C., \& Kerr, F.H. (1987). On Namia's fractional order Fourier transform. IMA. J. Appl. Math., 39(2), 159-175.

3. Alieva, T., Lopez, V., Agullo-Lopez, F., \& Almeida, L.B. (1994). The fractional Fourier transform in optical propagation problems. J. Mod. Opt., 41(5), 1037-1040.

4. Almeida, L.B. (1994). The fractional Fourier transform and time-frequency representation. IEEE Trans. Signal Process., 42, 3084-3091.

5. Castro, L.P., \& Zhang, B. (2005). Invertibility of convolution operators arising in problems of wave diffraction by a strip with reactance and Dirichlet conditions. $Z$. Anal. Anwend., 24, 545-560.

6. Durak, L., \& Aldirmaz, S. (2010). Adaptive fractional Fourier domain filtering. Signal Processing, 90 (4), 1188-1196.

7. Mendlovic, D., \& Ozaktas, H.M. (1993). Fractional Fourier transforms and their optical implementation. J. Opt. Soc. Amer. A, 10, 1875-1881.

8. Ozaktas, H.M., Arıkan, O., Kutay, M.A., \& Bozdağı, G. (1996). Digital computation of the fractional Fourier transform. IEEE Trans. Signal Process., 44, 2141-2150.

9. Ozaktas, H.M., \& Mendlovic, D. (1993). Fractional Fourier transforms and their optical implementation II. J. Opt. Soc. Amer. A, 10, 2522-2531.

10. Barshan, \& B. Ayrulu, B. (2002). Fractional Fourier transform pre-processing for neural networks and its application to object recognition. Neural Networks, 15(1), $131-140$.

11. Cui, D. (2009). Dual digital watermarking algorithm for image based on fractional Fourier transform. Proceedings of the Second Pacific-Asia Conference on Web Mining and Web-based Application (WMWA 09), Wuhan, China, June 6-7, 51-54.

12. Djurovic, I., Stankovic, S., \& Pitas, I. (2001). Digital watermarking in the fractional Fourier transformation domain. Journal of Network and Computer Applications, $24(2), 167-173$.

13. Ran, Q., Zhang, H., Zhang, J., Tang, L., \& Ma, J. (2009). Deficiencies of the cryptography based on multiple-parameter fractional Fourier transform. Opt. Lett., 34(11), 1729-1731.

14. Yu, F.Q., Zhang, Z.K., \& Xu, M.H. (2006). A digital watermarking algorithm for image based on fractional Fourier transform. Proceedings of the 2006 IEEE Conference on Industrial Electronics and Applications, Singapore, May 24-26, 1-5.

15. Ozaktas, H.M., Zalevsky, Z., \& Kutay, M.A. (2001) The fractional Fourier transform with applications in optics and signal processing. John Wiley, New York.

16. Cohen, L. (1989). Time-frequency distributions-A review. Proc. IEEE, 77(7), 941-981.

17. Hlawatsch, F., \& Bourdeaux-Bartels, F.G. (1992). Linear and quadratic timefrequency signal representations. IEEE Signal Processing Mag., 9(2), 21-67.

18. Erden, M.F., Kutay, M.A., \& Ozaktas, H.M. (1999). Applications of the fractional Fourier transform to filtering, estimation and restoration. Proceedings of the IEEEEURASIP Workshop on Nonlinear Signal and Image Processing (NSIP 99), Antalya, Turkey, 481-485.

19. Kutay, M.A., Ozaktas, H.M., Arikan, O., \& Onural, L. (1997). Optimal filtering in fractional Fourier domains. IEEE Trans. Signal Process., 45(5), 1129-1143.

20. Liu, S.-G., \& Fan, H.-Y. (2009). Convolution theorem for the three-dimensional entangled fractional Fourier transformation deduced from the tripartite entangled state representation. Teoret. Mat. Fiz., 161(3), 459-468.

21. Ozaktas, H.M., Barshan, B., \& Mendlovic, D. (1994). Convolution and filtering in fractional Fourier domains. Optical Review, 1(1), 15-16.

22. Singh, A.K., \& Saxena, R. (2012). On Convolution and Product Theorems for FRFT. Wireless Personal Communications, 65(1), 189-201.

23. Wei, D., Ran, Q., Li, Y., Ma, J., \& Tan, L. (2009). A convolution and product theorem for the linear canonical transform. IEEE Signal Processing Lett., 16(10), 853-856.

24. Zayed, A.I. (1998). A convolution and product theorem for the fractional Fourier transform. IEEE Signal Processing Lett., 5(4), 102-103. 
25. Almeida, L.B. (1997). Product and convolution theorems for the fractional Fourier transform. IEEE Trans. Signal Process. Lett., 4(1), 15-17.

26. Bing, D., Ran, T., \& Yue, W., (2006). Convolution theorems for the linear canonical transform and their applications. Sci. China Ser. F-Inform. Sci., 49 (5), 592-603.

27. Giang, B.T., Mau, N.V., \& Tuan, N.M. (2009). Operational properties of two integral transforms of Fourier type and their convolutions. Integral Equations Operator Theory, 65, 363-386.

28. Giang, B.T., Mau, N.V., \& Tuan, N.M. (2010). Convolutions for the Fourier transforms with geometric variables and applications. Math. Nachr., 283, 1758-1770.

29. Rudin, W. (1991) Functional Analysis. New York: McGraw-Hill. 